\title{
High expression of UBD correlates with epirubicin resistance and indicates poor prognosis in triple- negative breast cancer
}

\author{
Tao Han, ${ }^{1, *}$ Zhaozhe Liu, ${ }^{1, *}$ \\ Hengyu $\mathrm{Li}^{2},{ }^{2} *$ Wanqing \\ Xie, ${ }^{3, *}$ Ranran Zhang,' \\ Li Zhu, ${ }^{2}$ Fang Guo,' \\ Yaling Han, ${ }^{4}$ Yuan Sheng, ${ }^{2}$ \\ Xiaodong $\mathrm{Xie}^{1}$ \\ 'Department of Oncology, Cancer \\ Center of People's Liberation Army, \\ General Hospital of Shenyang Military \\ Region, Shenyang, People's Republic \\ of China; ${ }^{2}$ Department of Thyroid and \\ Breast Surgery, Changhai Hospital, \\ Second Military Medical University, \\ Shanghai, People's Republic of China; \\ ${ }^{3}$ Liaoning University of Traditional \\ Chinese Medicine, Shenyang, People's \\ Republic of China; ${ }^{4}$ Department of \\ Cardiology, Institute of Cardiovascular \\ Research of People's Liberation Army, \\ General Hospital of Shenyang Military \\ Region, Shenyang, People's Republic \\ of China \\ *These authors contributed equally \\ to this work
}

Correspondence: Xiaodong Xie Department of Oncology, Cancer Center of People's Liberation Army, General Hospital of Shenyang Military Region, 83 Wenhua Road, Shenyang, Liaoning I I 0840, People's Republic of China

Tel +86242885 6310

Fax +86242885 6310

Email doctor_xxd@163.com

Yuan Sheng

Department of Thyroid and Breast Surgery, Changhai Hospital, Second Military Medical University, I 68 Changhai Road, Shanghai, 200438, People's

Republic of China

$\mathrm{Tel}+862181875364$

Email crzs28I@tom.com
This article was published in the following Dove Press journal:

OncoTargets and Therapy

6 July 2015

Number of times this article has been viewed

Background: Triple-negative breast cancer (TNBC) is a heterogeneous subtype of breast cancer that is prone to recurrence and metastasis with worse prognosis. Epirubicin-based chemotherapy is of great importance for patients with TNBC, but resistance to epirubicin severely limits the application of this drug and this has emerged as a major problem in the treatment of TNBC. The ubiquitin protein $\mathrm{D}(U B D)$ molecule has often been considered a tumor oncogene, and has been shown to promote the recurrence and metastasis of malignant tumor cells. Since the role of UBD in epirubicin resistance and its prognostic value in TNBC have not been reported, the study reported here was designed to identify the epirubicin-resistance molecule and clarify the related biomarker for TNBC prognosis.

Methods: UBD plasmid was transfected into MDA-MB-231 cells, and the cells were exposed to epirubicin to observe the ability of UBD in epirubicin resistance. UBD expression was also detected in 78 breast cancer tissues by immunohistochemistry. Statistical methods were used to study the relationship between UBD expression and epirubicin resistance in TNBC treatment. Kaplan-Meier survival analysis was used to determine the correlation between UBD expression and TNBC patients' prognostic parameters.

Results: UBD expression was found increased in breast cancer tissues. Forced UBD expression was found to have a relationship with TNBC epirubicin resistance in vitro. High expression of UBD was found in TNBC, compared with in non-TNBC, and this played a positive role in epirubicin resistance and indicated the poor prognosis of TNBC treatment.

Conclusion: UBD may play an important role in epirubicin resistance in TNBC. UBD has the potential to be a novel biomarker in TNBC chemoresistance and may be a promising therapeutic target for TNBC patients.

Keywords: prognosis, chemoresistance, biomarker

\section{Introduction}

Breast cancer is a highly prevalent cancer in women around the world. ${ }^{1}$ According to gene expression, five main intrinsic molecular subtypes of breast cancer are known: luminal A, luminal B, human epidermal growth factor receptor 2 (HER2) enriched, basal like, and claudin low. ${ }^{2}$ Triple-negative breast cancer (TNBC) is defined by the lack of expression of the estrogen receptor, progesterone receptor, and HER $2 /$ neu. ${ }^{3}$ Due to the lack expression of hormone receptors and HER2, endocrine therapy and specific molecule target therapy are not available for TNBC. Further, TNBC is characterized by uncontrolled proliferation, and it easily invades and metastasizes to distant organ sites, such as the lung, liver, brain, and bone. ${ }^{4}$ Thus, TNBC patients frequently develop therapeutic resistance and rapid disease relapse, which bring a higher risk of death in these 
patients. Chemotherapy remains one of the most important anticancer methods in TNBC. Epirubicin is an antineoplastic agent belonging to the second-generation anthracycline antibiotics, which plays an antitumor role by binding to DNA, bursting and stabilizing the fragmentation process of doublestranded DNA. ${ }^{5}$ In clinic, epirubicin has been widely used in the treatment of breast, ovarian, bladder, lung, bronchus, pancreatic, stomach, esophagus, and liver cancers. ${ }^{4}$

The ubiquitin protein D (UBD) molecule belongs to the ubiquitin protein family, and the binding to its target protein will result in the protein degrading due to the proteasome. ${ }^{6}$ Through degradation of I kappa B alpha and the intrinsic immune activation induced by lipopolysaccharides (LPS), ${ }^{6}$ UBD participates in the process of maturing dendritic cells. $^{7}$ Therefore, previous studies have focused on its role in the immune system. Research has revealed the important role of UBD expression in liver cancer. However, as far as we are aware, little research has been conducted exploring the role of UBD expression in TNBC chemoresistance and its relationship with the prognosis of patients with TNBC.

In the study reported here, we established the role of UBD in epirubicin resistance by studying the TNBC cell line MDA-MB-231. Further, we found enhanced UBD expression in breast cancer tissue and the clinical-pathological significance of this. We also studied the expression of UBD and its correlation with progression-free survival (PFS) in epirubicin-treated TNBC patients, and found that UBD plays an important role in epirubicin resistance in TNBC.

\section{Materials and methods}

\section{Cell culture}

The human TNBC cell line MDA-MB-231 was obtained from the Type Culture Collection of the Chinese Academy of Sciences (Shanghai, People's Republic of China). Cells were cultured in Roswell Park Memorial Institute (RPMI) 1640 medium containing 10\% fetal bovine serum (FBS; Gibco ${ }^{\circledR}$, Thermo Fisher Scientific, Waltham, MA, USA), and cultured at $37^{\circ} \mathrm{C}$ in humidified atmosphere of $95 \%$ air $/ 5 \% \mathrm{CO}_{2}$.

\section{Plasmid}

The plasmid pcDNA3-UBD and the scramble pcDNA3 were provided by the Laboratory of Signal Transduction, Eastern Hepatobiliary Surgery Institute, Second Military Medical University, Shanghai, People's Republic of China.

\section{Proliferation assay}

Cell proliferation was measured by 3-(4,5-dimethylthiazol2-yl)-2,5-diphenyltetrazolium bromide (MTT) assay. Cells were seeded at a density of 6,000 cells per well in 96-well plates. After 24 hours, the cells were exposed to varied concentrations of epirubicin or equal volumes of sterile apyrogenic water for indicated time ( 0 hour, 36 hours, 72 hours, 96 hours).

Then the cells were incubated with $0.5 \mathrm{mg} / \mathrm{mL}$ MTT (Sigma-Aldrich Co, St Louis, MO, USA) reagent for 4 hours' incubation at $37^{\circ} \mathrm{C}$. Subsequently, the culture medium was removed and $150 \mu \mathrm{L}$ of dimethyl sulfoxide was added to each well. The formazan crystals were solubilized by gently shaking the plates for 10 minutes and the absorbance was measured at $570 \mathrm{~nm}$. Cell proliferation was calculated as a percentage of the control and the half maximal inhibitory concentration was determined from the dose-dependent growth inhibition data using GraphPad Prism (Version 5.01; GraphPad Software, Inc, San Diego, CA, USA).

\section{Invasion assay}

The invasive ability of cells was evaluated using 24-well Transwell ${ }^{\circledR}$ chambers containing an $8 \mu \mathrm{m}$ pore polycarbonate filter (Corning Incorporated, Corning, NY, USA) coated with Matrigel ${ }^{\mathrm{TM}}$ (BD Biosciences, San Jose, CA, USA). The bottom chamber was filled with $600 \mu \mathrm{L}$ standard medium containing $10 \%$ FBS. Cells were plated at a density of $5 \times 10^{4}$ in the upper chamber suspended in $200 \mu \mathrm{L}$ serum-free medium supplemented with $20 \mathrm{nM}$ Epirubicin. After incubation for 48 hours, the non-invaded cells were removed with a cotton-tip swab. Invaded cells that stuck to the lower surface of the membrane were fixed in methanol and stained with crystal violet. The cells were photographed and counted under a microscope at $200 \times$ magnification in six random fields from triplicate wells.

\section{Tissue specimens and patient information}

This study was approved by the Ethics Committee of the General Hospital of Shenyang Military Region. Tissue specimens were collected from 78 breast cancer patients treated in our hospital between 2006 and 2011. Pathological diagnosis was achieved by surgery. All the patients were recommended to receive epirubicin-based chemotherapy. Clinicopathologic staging was determined according to the AJCC Cancer Staging Manual criteria. ${ }^{8}$ Tissue specimens were fixed in $4 \%$ paraformaldehyde and embedded in paraffin for immunohistochemistry (IHC).

Detailed clinicopathologic characteristics of the patients are summarized in Table 1.

\section{Immunohistochemistry and analysis}

Immunohistochemical analysis of tumor sections was performed using the anti-UBD antibody. Briefly, the sections 
Table I Clinical pathologic characteristics

\begin{tabular}{|c|c|c|c|}
\hline Variable & UBD high expression $(n=39)$ & UBD low expression $(n=39)$ & $P$ \\
\hline Median age, years (range) & $48(37-69)$ & $51(34-70)$ & 0.470 \\
\hline \multicolumn{4}{|l|}{ Sex, $\mathrm{n}$} \\
\hline Female & 39 & 39 & \\
\hline \multicolumn{4}{|l|}{ Smoking, n (\%) } \\
\hline Yes & $5(12.80)$ & $7(17.90)$ & 0.530 \\
\hline No & $34(87.20)$ & $32(82.10)$ & \\
\hline \multicolumn{4}{|l|}{ Alcohol history, n (\%) } \\
\hline Yes & $7(8.97)$ & $6(7.69)$ & 0.760 \\
\hline No & $32(91.03)$ & $33(92.31)$ & \\
\hline \multicolumn{4}{|l|}{ Tumor stage, n (\%) } \\
\hline $\mathrm{TI} / 2$ & $33(84.60)$ & $36(92.30)$ & 0.290 \\
\hline $\mathrm{T} 3 / 4$ & $6(15.40)$ & $3(7.70)$ & \\
\hline \multicolumn{4}{|l|}{ Nodal stage, n (\%) } \\
\hline No/I & $26(66.70)$ & $30(76.90)$ & 0.310 \\
\hline$N 2 / 3$ & $13(33.30)$ & $9(23.10)$ & \\
\hline \multicolumn{4}{|l|}{ Triple negative,* n (\%) } \\
\hline Yes & $21(53.80)$ & $7(17.90)$ & 0.001 \\
\hline No & $18(46.20)$ & $32(82.10)$ & \\
\hline \multicolumn{4}{|l|}{ AJCC stage, $n(\%)$} \\
\hline I-II & $20(51.30)$ & $21(53.80)$ & 0.820 \\
\hline III & $19(48.70)$ & $18(46.20)$ & \\
\hline \multicolumn{4}{|l|}{ Tumor diameter, $\mathrm{n}(\%)$} \\
\hline$>5 \mathrm{~cm}$ & $9(23.10)$ & $6(15.40)$ & 0.390 \\
\hline$<5 \mathrm{~cm}$ & $30(76.90)$ & $33(84.60)$ & \\
\hline
\end{tabular}

Note: *Results of the analysis $(P<0.05)$ are statistically significant (chi-square test).

Abbreviations: AJCC, American Joint Committee on Cancer; UBD, ubiquitin protein D.

were incubated with the primary antibody at $4^{\circ} \mathrm{C}$ overnight and then with horseradish peroxidase-conjugated secondary antibody at $37^{\circ} \mathrm{C}$ for 30 minutes. The sections were finally incubated with diaminobenzidine and counterstained with hematoxylin for detection. Assessment of the staining was based on the percentage of positively stained cells and the nuclear staining intensity evaluated by three pathology doctors. The procedure of human sample collection was approved by the ethical committee of the hospital.

\section{Real-time polymerase chain reaction}

Quantitative polymerase chain reaction (PCR) was performed using an Applied Biosystems SYBR ${ }^{\circledR}$ Green PCR Kit and Applied Biosystems 7900HT Fast Real-Time PCR System (both Thermo Fisher Scientific). The mRNA level of specific genes was normalized against $18 \mathrm{~S}$. The primer sequences used in this study are listed in Table 2.

\section{Western blot}

The cell extract was separated on polyacrylamide-sodium dodecyl sulfate gels, transferred, and probed with a specific primary antibody. The protein band, specifically bound to the primary antibody, was detected using an IRDye ${ }^{\circledR}$
800CW-conjugated secondary antibody and LI-COR imaging system (LI-COR Biosciences, Lincoln, NE, USA).

\section{Statistical analysis}

All data in this study were analyzed with SPSS software (v 18.0; IBM Corporation, Armonk, NY, USA). Reports were presented as mean values \pm standard error of the mean. The difference between two groups or paired samples was analyzed by Student's $t$-test. The differences of quantitative variables between groups were analyzed by one-way analysis of variance. Correlation analysis was performed using Pearson's correlation test. Survival curves were calculated using the Kaplan-Meier method and compared with a log-rank test. All statistical differences were two-sided, and $P<0.05$ was considered statistically significant.

Table 2 Sequence of primers for real-time polymerase chain reaction

\begin{tabular}{ll}
\hline Primer & Sequence $\left(\mathbf{5}^{\prime}\right.$ to $\mathbf{3}^{\prime}$ ) \\
\hline UBD forward primer & TCATATGGGTTGGCATCAAA \\
UBD reverse primer & TGTCTGCAGAGATGGCTCC \\
I8s forward primer & CGGCTACCACATCCAAGGAA \\
I8s reverse primer & GCTGGAATTACCGCGGCT \\
\hline
\end{tabular}

Abbreviation: UBD, ubiquitin protein $\mathrm{D}$. 


\section{Results}

High expression of UBD enhanced the epirubicin resistance in TNBC cell line

To identify the effect of UBD on epirubicin resistance in a TNBC cell line, MDA-MB-231 cells were transfected with UBD plasmid and the control scramble (Figure 1A). To determine the concentration of the epirubicin effect on the MDAMB-231 cells, the dose-dependent growth inhibition data of MDA-MB-231 were evaluated and results show that $20 \mathrm{nM}$ is close to the cell half maximal inhibitory concentration (Figure 1B). As shown in Figure 1B, UBD overexpression remarkably suppressed the proliferation of MDA-MB-231 cells treated with $20 \mathrm{nM}$ epirubicin. Invasion chamber assay was used to investigate whether UBD upregulation exerted inhibitory effects on cell migration and invasion. As shown in Figure $1 \mathrm{C}$, the migration ability of MDA-MB-231 cells treated with $20 \mathrm{nM}$ epirubicin was significantly enhanced by highly expressed UBD.

\section{UBD expression was elevated in breast cancer and correlated with TNBC}

To explore the function of UBD expression in breast cancer, we examined UBD expression in 78 human breast cancer tissues. As shown in Figure 2A, UBD was significantly increased in breast cancer tissues compared with in the corresponding tissues, as determined by immunostaining analysis. Of note, breast cancer with poor prognosis exhibited higher expression levels of UBD (Figure 2B). Further, it was revealed that the
A

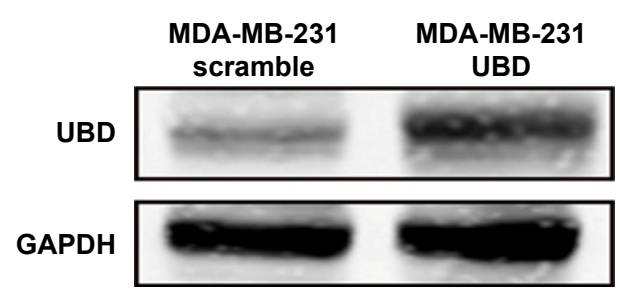

C

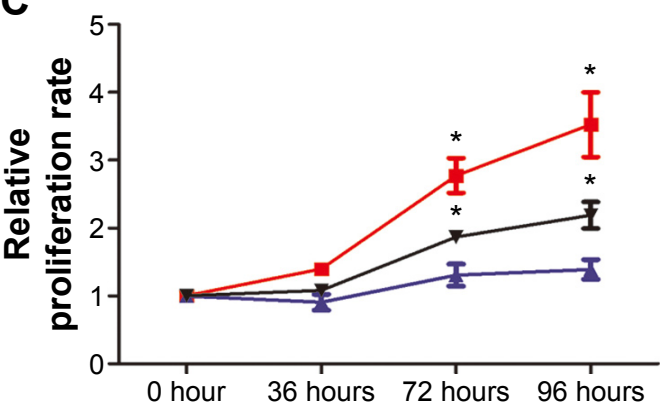

D
B

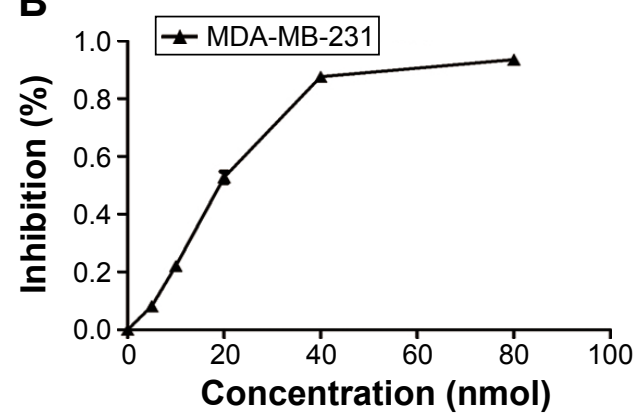

$\rightarrow$ MDA-MB-231 treated by DMSO
$\rightarrow$ MDA-MB-231 scramble treated by epirubicin
$\rightarrow$ MDA-MB-231 UBD treated by epirubicin
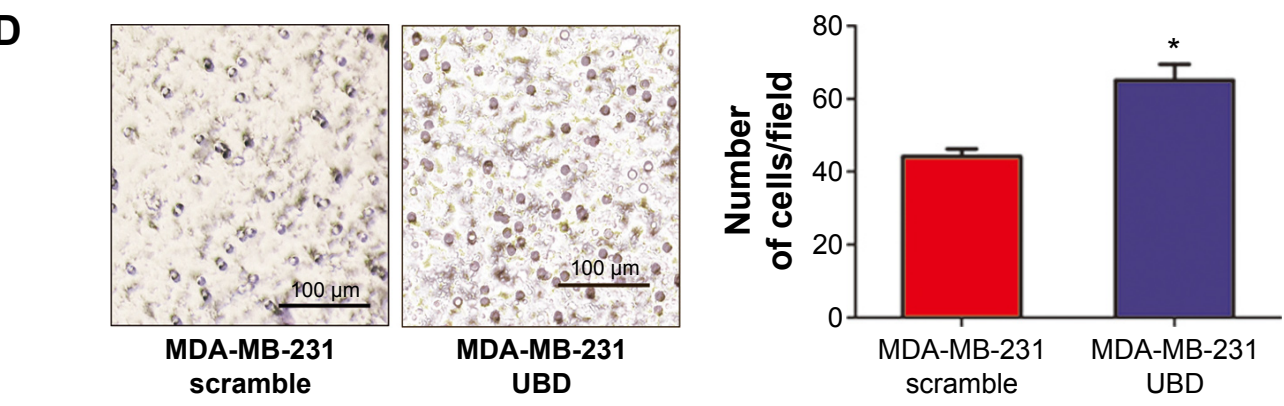

Figure I Ubiquitin protein D (UBD) overexpression enhanced the resistance of epirubicin in triple-negative breast cancer cell lines. (A) Representative Western blot showing the overexpression of UBD in the MDA-MB-23I cell line transfected by UBD plasmid. (B) The inhibition rate of MDA-MB-23I cells treated with different concentrations of epirubicin by MTT, and the half maximal inhibitory concentration of MDA-MB-23I is about $20 \mathrm{nM}$. (C) The relative proliferation of UBD and its control cells was evaluated by MTT assay in MDA-MB-23I cell lines overexpressing UBD and their control plasmids treated with epirubicin (20 nM). The relative proliferation of blank triple-negative breast cancer cells, which were not treated with a chemotherapy drug, was also measured by MTT. (D) Migration assays were undertaken in MDA-MB-23 I cells overexpressing UBD and their control plasmids treated with epirubicin $(20 \mathrm{nM})$.

Notes: The lines above the bars indicated Standard Deviation. The asterisk $\left(^{*}\right)$ indicated $P<0.05$. The length of the scale bar indicated I00 $\mu \mathrm{m}$.

Abbreviations: DMSO, dimethyl sulfoxide; GAPDH, glyceraldehyde 3-phosphate dehydrogenase; MTT, 3-(4,5-dimethylthiazol-2-yl)-2,5-diphenyltetrazolium bromide. 
A

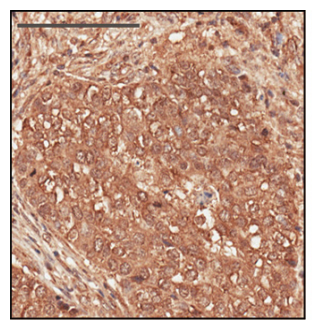

Breast cancer

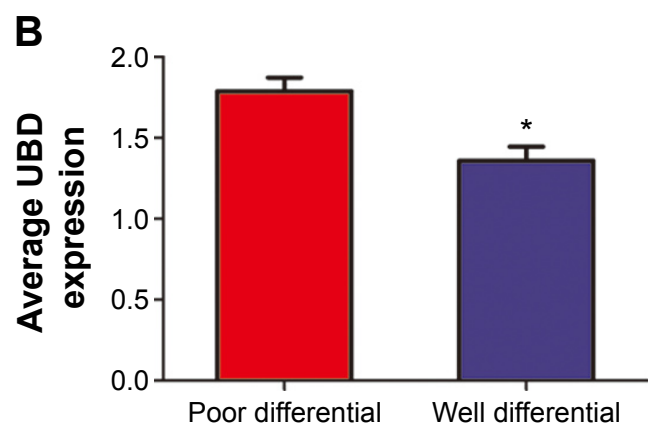

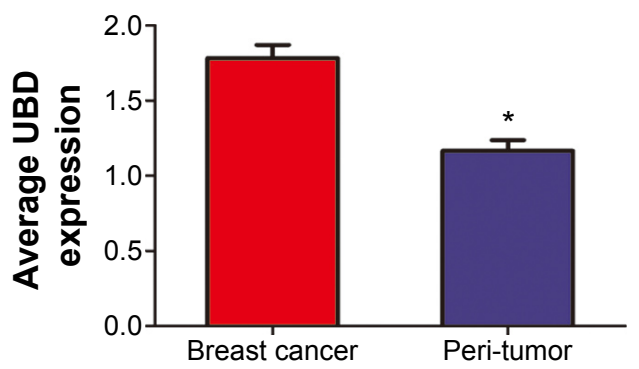

C

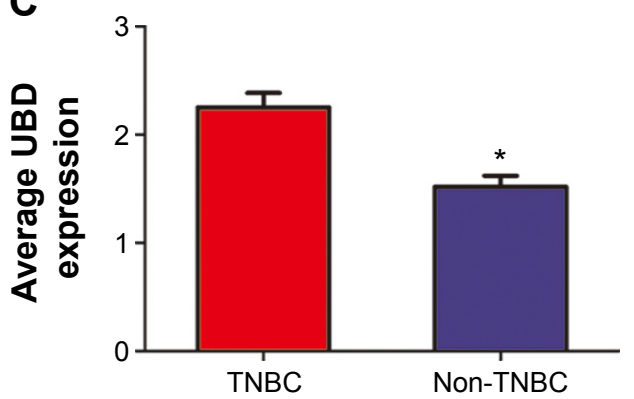

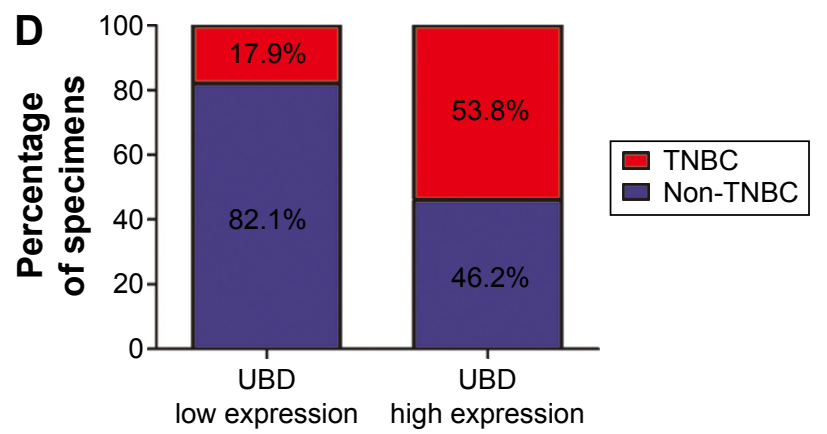

Figure 2 Ubiquitin protein $D(U B D)$ expression is elevated in breast cancer and has a closed relationship with triple-negative breast cancer (TNBC). (A) The average UBD density in 78 paired breast cancer tissue samples was measured using immunohistochemical (IHC) staining - a representative view is shown. (B) Comparison of UBD expression in 78 different differentiated human breast cancer tissues using IHC staining. (C) Comparison of UBD expression in TNBC and non-TNBC by IHC staining. (D) The percentage of TNBC and non-TNBC in UBD overexpression and low expression tissues.

Notes: (A) Scale bar: $100 \mu \mathrm{m}$. (B, C) Data were analyzed by $t$-test $(* P<0.05)$.

majority of patients with a high expression of UBD were prone to have TNBC (Figure 2C), and UBD was detected at higher amounts in TNBC than in non-TNBC (Figure 2D). Based on immunostaining results, the 78 human breast cancer patient tissues were divided into two groups: high UBD expression group ( $n=39)$ and low UBD expression group $(n=39)$. Tissues in the UBD high group were prone to having TNBC compared with those in the non-TNBC group $(P<0.05$; Table 1).

\section{High expression of UBD indicated the poor prognosis of TNBC patients treated with epirubicin}

In our cohort, 28 patients were diagnosed as having TNBC by pathology. Twenty-one patients had a higher UBD expression and seven patients had a lower UBD expression. Epirubicin-based adjuvant chemotherapy was administrated to the patients and the PFS was observed. In patients with a higher expression of UBD, the median PFS was 23 months. In contrast, the patients with a lower expression of UBD had a median PFS of 36 months. A trend toward poor PFS was found in patients with higher expression levels of UBD compared with those with lower UBD expression levels $(P<0.05$; Figure $3 \mathrm{~A}$ ). This possibly implies that a high expression of UBD enhanced the chemotherapy resistance in epirubicinbased adjuvant chemotherapy and mediated the poor prognosis of TNBC.

\section{Discussion}

Breast cancer accounts for $30 \%-40 \%$ of malignant tumors in women. It is responsible for the second highest rate of cancer-related mortality in the USA, with an estimated 39,620 deaths in 2013.9,10 Along with the public's great concern, 


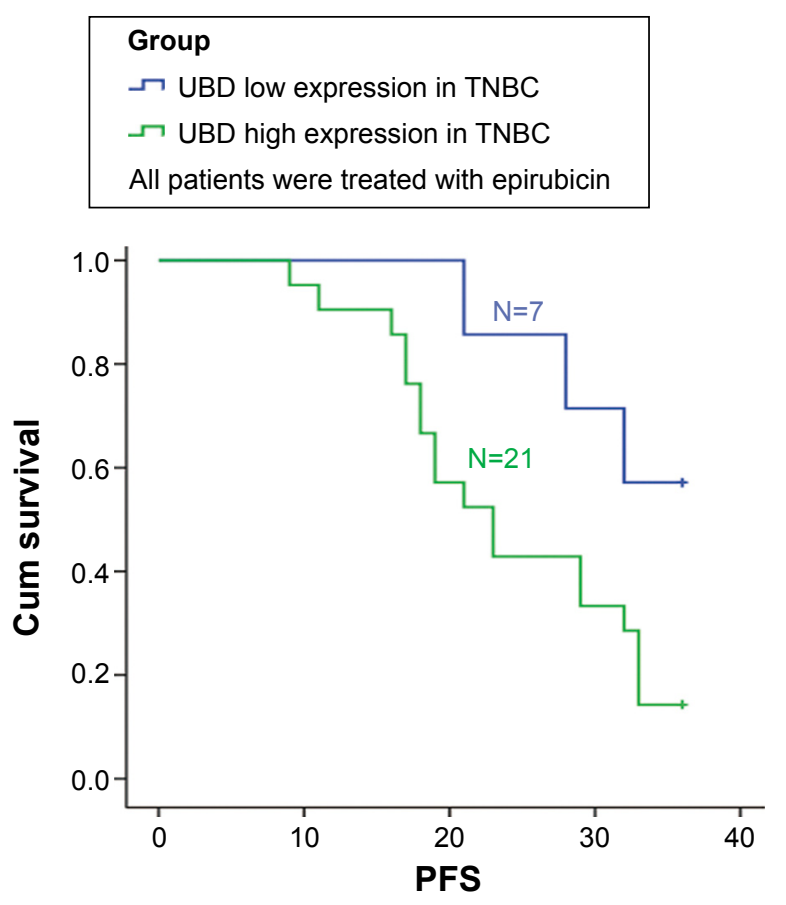

Figure 3 Ubiquitin protein $D(U B D)$ expression has a closed relationship with the prognosis of triple-negative breast cancer (TNBC) patients treated with epirubicin chemotherapy. Disease-free survival analyses of 28 TNBC patients treated with epirubicin were compared between the UBD low expression (below the median value) and UBD high expression (above the median value) groups.

Note: The units for the values on $\mathrm{x}$-axis indicated months.

Abbreviations: PFS, progression-free survival; cum, cumulative.

studies have confirmed that the prognosis is associated with the molecular subtypes of patients with breast cancer. TNBC, a heterogeneous subtype of breast cancer, is particularly prone to recurrence, metastasis, and chemotherapy resistance. So far, no effective molecular targeted drug has been made available for TNBC. Surgical resection and chemotherapy are the therapeutic means in clinic. According to the National Comprehensive Cancer Network guideline, epirubicinbased adjuvant chemotherapy is recommended and the curative effect will directly influence the patient's prognosis. ${ }^{11}$ However, resistance to epirubicin is very common in TNBC patients and the mechanism is still unclear. Gyémánt et al explored the synergistic effect between flavonoids and epirubicin on multidrug-resistant cancer cells. ${ }^{12}$ They found that chrysin and amorphigenin synergistically enhanced the effect of epirubicin on the mouse lymphoma cell line and formononetin did the same on the MDA-MB-231 cell line.

UBD has been reported a tumor oncogene that promotes tumor cells' malignant biological behaviors, such as recurrence and metastasis, via multiple signaling pathways including phosphatidylinositol-4,5-bisphosphate 3-kinase $(\mathrm{PI} 3 \mathrm{~K}) /$ protein kinase B (Akt), Wnt/beta-catenin, and nuclear factor kappa-B. ${ }^{13-16}$ Given the positive connection between UBD activation and tumor progression, it was predicted to be of importance in breast cancer development. However, there have been limited reports in support of this theory. In the study reported here, in vitro experiments were conducted and clinical breast cancer samples were involved. We found that UBD expression correlated with epirubicin resistance in MDA-MB-231 cells and that the level of UBD was significantly increased in breast cancer tissues. Also, we found that the majority of patients with high expression levels of UBD were prone to be hormonereceptor negative, Her2 negative, and to have cancer with a poor prognosis. Further analysis revealed that the TNBC patients with an overexpression of UBD obtained worse clinical benefit and a higher recurrence rate from epirubicinbased chemotherapy than those with a low level of UBD expression. The findings imply that UBD overexpression enhanced the chemotherapy resistance of epirubicin-based adjuvant chemotherapy.

\section{Conclusion}

Epirubicin-based chemotherapy is of great importance in improving the prognosis of patients with TNBC, but the resistance to epirubicin severely limits the application of this drug. Identifying the epirubicin-resistance molecule and clarifying the related biomarker for TNBC prognosis could enable the development of successful strategies to circumvent this issue. In the study reported here, we demonstrated that UBD upregulation is involved in the process of epirubicin resistance in TNBC patients, which was confirmed by experiments in vitro. It is worth exploring whether inhibition of UBD might be a potential therapeutic approach in the treatment of epirubicin resistance in TNBC.

\section{Acknowledgments}

The authors thank Dr Di Chen for his kind help and guidance. This work was supported by the Key Project of the National 12th Five-Year Research Program of China (number 2012ZX0903016-002). The funders had no role in the design of the study, data collection and analysis, decision to publish, or preparation of the manuscript for publication.

\section{Disclosure}

The authors declare no conflicts of interest in this work.

\section{References}

1. Parkin DM, Bray F, Ferlay J, Pisani P. Estimating the world cancer burden: Globocan 2000. Int J Cancer. 2001;94(2):153-156.

2. Kristensen VN. Divide and conquer: the genetic basis of molecular subclassification of breast cancer. EMBO Mol Med. 2011;3(4):183-185. 
3. Maire V, Némati F, Richardson M, et al. Polo-like kinase 1: a potential therapeutic option in combination with conventional chemotherapy for the management of patients with triple-negative breast cancer. Cancer Res. 2013;73(2):813-823.

4. Anders CK, Carey LA. Biology, metastatic patterns, and treatment of patients with triple-negative breast cancer. Clin Breast Cancer. 2009;9 Suppl 2:S73-S81.

5. Forrest RA, Swift LP, Evison BJ, et al. The hydroxyl epimer of doxorubicin controls the rate of formation of cytotoxic anthracycline-DNA adducts. Cancer Chemother Pharmacol. 2013;71(3):809-816.

6. Hofmann K. Ubiquitin-binding domains and their role in the DNA damage response. DNA Repair (Amst). 2009;8(4):544-556.

7. Bates EE, Ravel O, Dieu MC, et al. Identification and analysis of a novel member of the ubiquitin family expressed in dendritic cells and mature B cells. Eur J Immunol. 1997;27(10):2471-2477.

8. Edge SB, Byrd DR, Compton CC, Fritz AG, Greene FL, Trotti A, editors. AJCC cancer staging manual (7th ed). New York, NY: Springer; 2010.

9. Toriola AT, Colditz GA. Trends in breast cancer incidence and mortality in the United States: implications for prevention. Breast Cancer Res Treat. 2013;138(3):665-673.
10. Pujol H. Trends in breast cancer incidence, survival, and mortality. Lancet. 2000;356(9229):591-952; author reply 593.

11. Joerger M, Ess S, Dehler S, et al. A population-based study on the patterns of use of different chemotherapy regimens in Swiss patients with early breast cancer. Swiss Med Wkly. 2012. 142:w13571.

12. Gyémánt $\mathrm{N}$, Tanaka M, Antus $\mathrm{S}$, et al. In vitro search for synergy between flavonoids and epirubicin on multidrug-resistant cancer cells. In Vivo. 2005;19(2):367-374.

13. Lee CG, Ren J, Cheong IS, et al. Expression of the FAT10 gene is highly upregulated in hepatocellular carcinoma and other gastrointestinal and gynecological cancers. Oncogene. 2003;22(17):2592-2603.

14. Liu L, Dong Z, Liang J, et al. As an independent prognostic factor, FAT10 promotes hepatitis B virus-related hepatocellular carcinoma progression via Akt/GSK3 $\beta$ pathway. Oncogene. 2014;33(7):909-920.

15. Gong P, Canaan A, Wang B, et al. The ubiquitin-like protein FAT10 mediates NF-kappaB activation. J Am Soc Nephrol. 2010;21(2): 316-326.

16. Yuan $\mathrm{R}$, Wang $\mathrm{K}, \mathrm{Hu} \mathrm{J}$, et al. Ubiquitin-like protein FAT10 promotes the invasion and metastasis of hepatocellular carcinoma by modifying $\beta$-catenin degradation. Cancer Res. 2014;74(18):5287-5300.
OncoTargets and Therapy

\section{Publish your work in this journal}

OncoTargets and Therapy is an international, peer-reviewed, open access journal focusing on the pathological basis of all cancers, potential targets for therapy and treatment protocols employed to improve the management of cancer patients. The journal also focuses on the impact of management programs and new therapeutic agents and protocols on

\section{Dovepress}

patient perspectives such as quality of life, adherence and satisfaction. The manuscript management system is completely online and includes a very quick and fair peer-review system, which is all easy to use. Visit http://www.dovepress.com/testimonials.php to read real quotes from published authors. 